\title{
Is There a Role for Rituximab in the Treatment of Spondyloarthritis and Psoriatic Arthritis?
}

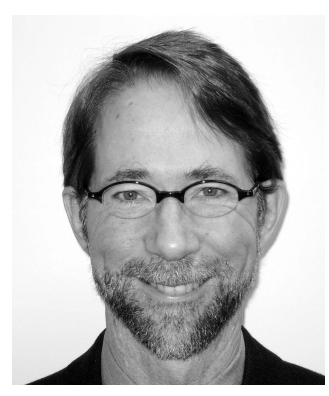

There is a significant unmet need for effective therapy options for patients with spondyloarthritis (SpA) who are primary or secondary inadequate responders to anti-tumor necrosis factor- $\alpha$ (anti-TNF- $\alpha$ ) medications, or who have experienced serious adverse effects or have contraindications to these agents. Although traditional oral disease-modifying drugs (DMARD) such as methotrexate, leflunomide, and sulfasalazine have not shown efficacy in the spondylitis domain they have the potential to be effective for more peripheral domains of synovitis and enthesitis, although here, too, patients may not gain adequate benefit or may be intolerant ${ }^{1}$. For rheumatoid arthritis (RA), there is a growing repertoire of approved agents with different mechanisms of action that have proven efficacy. These agents include the $\mathrm{T}$ cell costimulatory blockade agent abatacept, the B cell ablator rituximab, and the interleukin 6 (IL-6) receptor inhibitor tocilizumab. On the surface, it is tempting to think that these same agents will be efficacious for the various clinical manifestations of $\mathrm{SpA}$ - spondylitis, enthesitis, dactylitis, peripheral synovitis - and, in certain patients, nonmusculoskeletal manifestations such as psoriasis, colitis, and uveitis, since many of the same cellular and cytokine pathways of inflammation are present in SpA. Yet with closer investigation, it is now known that there are important differences in inflammatory mechanisms between RA and SpA such that it is not a given that a drug effective in RA will automatically be effective in $\mathrm{SpA}$, thus necessitating clinical trials to assess the efficacy of these drugs in SpA.

In this issue of The Journal, Wendling, et al describe 26 SpA patients treated with rituximab enrolled in the French AIR (Autoimmunity and Rituximab) registry: 10 with ankylosing spondylitis (AS), 7 with undifferentiated SpA, and 9 with psoriatic arthritis (PsA) ${ }^{2}$. Twenty-three patients had been treated with anti-TNF agents, mean number 2.3. Nineteen patients received one cycle of rituximab and mean number of courses was 1.5 among the 26 . Clinical outcomes were reported for 23 patients, and of these "efficacy was noted" in $11(47.8 \%)$, as judged by change in the Bath Ankylosing Spondylitis Disease Activity Index (BASDAI) or improvement of the swollen joint count. Of these 11, 7 had a predominantly axial presentation and 3 reached a BASDAI-50 response. In 4 cases with predominantly peripheral manifestation (2 PsA and 2 peripheral $\mathrm{SpA}$ ), mean swollen joint count improved from 4 to 1 . Safety observations were minimal, although it is notable that psoriasis worsened in 2 patients. Although it was not a controlled trial, the observations suggest that there may have been clinically meaningful improvement of musculoskeletal symptoms and signs in a small number of patients; but in sum, the authors conclude that B lymphocyte ablation may not be an appropriate target for SpA patients in general.

Should rituximab be included in a list of possible off-label medicines to consider in patients who have not responded to or cannot take anti-TNF, traditional DMARD, and nonsteroidal antiinflammatory drugs (NSAID)? To address this question, let's look at what we know about SpA pathophysiology vis à vis the potential benefit of $\mathrm{B}$ cell ablation and what other clinical data exist regarding rituximab in SpA.

The pathophysiology and clinical features of SpA and PsA are distinct from RA as evidenced by differing clinical features such as spondylitis, enthesitis, dactylitis, distinctive gut and eye inflammation, relatively greater role for innate compared to adaptive immunity, and unique cellular and cytokine profiles in bone, enthesial and synovial pathology ${ }^{3,4}$. Regarding B cell biology pertinent to rituximab therapy, in synovial membrane studies the number of $\mathrm{B}$ cells and plasma cells is lower in $\mathrm{SpA}$ than in $\mathrm{RA}^{5}$. In general, patients with $\mathrm{SpA}$ do not produce autoantibodies such as rheumatoid factor and anticitrullinated protein antibodies, important products of B cells in RA. Although B cell aggregates have been noted in PsA synovial membranes $^{6}$, in general the synovial membrane of SpA and PsA patients is devoid of lymphoid aggregation. The same holds

See Rituximab treatment for spondyloarthritis, page 2327 
true for psoriatic skin lesions. SpA may develop in the absence of $\mathrm{B}$ cells ${ }^{7}$. It has been suggested that rituximab treatment may lead to the development of psoriasis, although the mechanism for such a phenomenon is unknown $^{8,9,10,11}$. Nonetheless, B cells may play a role in antigen presentation in $\mathrm{SpA}$, even if synovial, skin, gut, and eye resident $\mathrm{B}$ cells are low in number, thus the need for clinical trials to assess the potential efficacy of a B cell ablator such as rituximab.

Case reports and open-label studies have demonstrated modest clinical benefit in some patients with SpA and PsA. Song, et al treated 20 patients with AS, half of whom were naive to anti-TNF therapy ${ }^{12}$. In 10 of these subjects who had previously been treated with anti-TNF therapy, there was no clear response at 24 weeks [Assessment of Spondyloarthritis International Society (ASAS20) response in 30\%, no ASAS50 response], but in 10 subjects who were naive to anti-TNF therapy, more significant efficacy was seen $(50 \%$ ASAS response and $30 \%$ in partial remission). The reason for this difference, other than that the anti-TNF-naive group were less "treatment refractory," is unknown. A similar finding was demonstrated by Mease, et al in 20 patients with PsA treated with rituximab in an open-label study ${ }^{13}$. In the overall group, $30 \%$ had an American College of Rheumatology (ACR20) response and 30\% had a Psoriasis Area and Severity Index (PASI50) response (nonresponder imputation analysis), suggesting modest response in the joints and skin. Six patients naive to anti-TNF therapy in that study demonstrated higher ACR and PASI responses, consistent with being less refractory to treatment or other unknown factors. Skin biopsies were obtained in 5 patients. Despite a paucity of B cells in lesional or nonlesional biopsies, elevated levels of IL-1ß, IL-8, and interferon- $\gamma$ in lesional skin were all significantly decreased at Week 8 after treatment, whereas TNF- $\alpha$ levels were unchanged, suggesting that immunological activity affecting some proinflammatory cytokines was occurring elsewhere (regional lymph nodes?). Jimenez-Boj, et al treated 9 PsA patients with rituximab, 8 of whom were naive to anti-TNF therapy, and noted a mean improvement of DAS28 from 6.2 to $4.9^{14}$. They also noted a Psoriatic Arthritis Response Criteria response in 5 patients, although it should be noted that this measure frequently demonstrates high placebo response. No improvement in skin lesions was noted. Together, these results suggest some patients with $\mathrm{SpA}$ and PsA may experience modest improvement in musculoskeletal symptoms and signs. Little skin benefit has been seen, despite decreases of some inflammatory cytokines in lesional skin biop$\operatorname{sies}^{13}$. The best results of rituximab treatment have been demonstrated in patients naive to anti-TNF therapy, whereas the greatest need is in patients who have had inadequate response to anti-TNF therapy. There are patients who may have a relative contraindication to anti-TNF therapy who, in light of this efficacy data, may be considered candidates for rituximab therapy. A case in point is a patient from our own practice, an individual with PsA and a recent history of lymphoma (for which she had received rituximab along with other medications), whose oncologist preferred the patient not receive anti-TNF therapy. Off-label use of rituximab yielded good musculoskeletal benefit. The few psoriasis skin lesions she demonstrated did not change significantly, but this was of minor import to her. Safety issues, including the potential risk of progressive multifocal leukoencephalopathy, were considered acceptable by this patient, who had previously been treated with rituximab for her lymphoma. A further point is that in larger open-label studies, rituximab use has not appeared to result in significant new onset or flares of psoriasis, although as noted, case reports have suggested such a potential association.

In summary, several points can be made: (1) There is an unmet medical need for medicines with a different mechanism than TNF inhibition, for patients with SpA or PsA who do not benefit from or are intolerant to TNF inhibitors. (2) One cannot assume that all non anti-TNF treatments for RA will work adequately in patients with SpA and PsA, given important differences in pathophysiology of these conditions, thus necessitating exploratory clinical trials. (3) Modest benefit for axial and peripheral symptoms of SpA and PsA has been noted in some patients in open-label exploratory studies of rituximab, as in the current study by Wendling, et al, particularly in patients who are anti-TNF-naive. (4) Risk-benefit evaluation of select SpA-PsA patients may support use of rituximab in some instances. (5) Controlled studies will be needed for more definitive understanding of the effect of rituximab in SpA-PsA.

PHILIP J. MEASE, MD,

Swedish Medical Center, University of Washington, Seattle, Washington, USA

Address correspondence to Dr. P.J. Mease, Seattle Rheumatology Associates, 1101 Madison Street, Suite 1000, Seattle, WA 98104. E-mail:pmease@philipmease.com

\section{REFERENCES}

1. van der Heijde D, Sieper J, Maksymowych WP, Dougados M, Burgos-Vargas R, Landewe R, et al. 2010 Update of the international ASAS recommendations for the use of anti-TNF agents in patients with axial spondyloarthritis. Ann Rheum Dis 2011;70:905-8.

2. Wendling D, Dougados M, Berenbaum F, Brocq O, Schaeverbeke T, Mazieres B, et al. Rituximab treatment for spondyloarthritis. A nationwide series: data from the AIR registry of the French Society of Rheumatology. J Rheumatol 2012;39:2327-31.

3. Mease PJ. Psoriatic arthritis: Update on pathophysiology, assessment and management. Ann Rheum Dis 2011;70:177-84.

4. Ronneberger M, Schett G. Pathophysiology of spondyloarthritis. Curr Rheumatol Rep 2011;13:416-20.

5. De Rycke L, Kruithof E, Vandooren B, Tak PP, Baeten D. Pathogenesis of spondyloarthritis: Insights from synovial membrane studies. Curr Rheumatol Rep 2006;8:275-82. 
6. Canete JD, Santiago B, Cantaert T, Sanmarti R, Palacin A, Celis R, et al. Ectopic lymphoid neogenesis in psoriatic arthritis. Ann Rheum Dis 2007;66:720-6.

7. Baeten D, Kruithof E, Breban M, Tak PP. Spondylarthritis in the absence of B lymphocytes. Arthritis Rheum 2008;58:730-3.

8. Dass S, Vital EM, Emery P. Development of psoriasis after B cell depletion with rituximab. Arthritis Rheum 2007;56:2715-8.

9. Mielke F, Schneider-Obermeyer J, Dorner T. Onset of psoriasis with psoriatic arthropathy during rituximab treatment of non-Hodgkin lymphoma. Ann Rheum Dis 2008;67:1056-7.

10. Hardcastle SA, Gibbs S, Williamson L. Atypical psoriasis following rituximab for rheumatoid arthritis. J Rheumatol 2012;39:1303-4.

11. Markatseli TE, Kaltsonoudis ES, Voulgari PV, Zioga A, Drosos AA. Induction of psoriatic skin lesions in a patient with rheumatoid arthritis treated with rituximab. Clin Exp Rheumatol 2009; 27:996-8
12. Song IH, Heldmann F, Rudwaleit M, Listing J, Appel H, Braun J, et al. Different response to rituximab in tumor necrosis factor blocker-naive patients with active ankylosing spondylitis and in patients in whom tumor necrosis factor blockers have failed: A twenty-four-week clinical trial. Arthritis Rheum 2012;62:1290-7.

13. Mease P, Kavanaugh A, Genovese M, Ritchlin C, Rosengren S, Quistberg A, et al. Rituximab in psoriatic arthritis provides modest clinical improvement and reduces expression of inflammatory biomarkers in skin lesions [abstract]. Arthritis Rheum 2010;62 Suppl:S818.

14. Jimenez-Boj E, Stamm TA, Sadlonova M, Rovensky J, Raffayova H, Leeb B, et al. Rituximab in psoriatic arthritis: An exploratory evaluation. Ann Rheum Dis 2012 July 24. (Epub ahead of print).

J Rheumatol 2012;39:2235-7; doi:10.3899/jrheum.121149 